Conclusions Taken together, these results exclude possible imprinting in $2 q$ as a cause of RSS in this child and suggest an autosomal recessive mutation which was unmasked by the segmental maternal isodisomic abnormality. Next Generation Sequence analysis of chromosome $2 \mathrm{q}$ regions of homozygosity identified in this child is underway and will most likely identify another novel RSS locus.

\section{SERIAL CYTOKINE EXPRESSIONS IN INFANTS WITH INCONTINENTIA PIGMENTI}

doi:10.1136/archdischild-2012-302724.0247

'SL Liao, ${ }^{2}$ SH Lai, ${ }^{2}$ WI Lee. 'Pediatrics, Chang Gung Memorial Hospital, Keelung, Keelung City; ${ }^{2}$ Pediatrics, Chang Gung Memorial Hospital, Taoyuan, Taiwan R.O.C.

Background NF-kB dysfunction resulting from NEMO (NFkappaB essential modulator) mutation can lead to significant alterations in cytokine production. However, little is known about changes in the expression of downstream molecules in patients with incontinentia pigmenti (IP).

Objective This study aims to investigate serial cytokine expressions during the first 2 years of life in young infants with IP, the period in which skin inflammation and morphological changes are most significant.

Methods Gene analysis was performed for the two neonates with IP. Peripheral mononuclear cells were obtained shortly after birth and successively at a 6-month interval up to the age of two years. Levels of TNF-a and IL- 6 were analyzed with ELISA before and after stimulating with TLR ligands.

Results The male patient had normal NEMO allele. His cytokine level, although initially lower, had returned to a level comparable with those of controls at 12 months of age. The female infant had a mutated NEMO gene. Her baseline TNF-a level was significantly higher than those of the control subjects at birth and remained high by 6 months of age. All cytokine responses had decreased significantly by 2 years of age, the time in which all vesicular skin lesions had resolved.

Conclusion This is the first report that demonstrates serial changes of cytokine profiles in humans with IP. This study showed that in the presence of NEMO mutation, alteration of cytokine production was remarkable during the first year of life, which may account for the prominent inflammatory changes in skin morphology.

\section{NOVEL MUTATION CAUSING GLUCOSE 1 TRANSPORTER DEFICIENCY SYNDROME}

doi:10.1136/archdischild-2012-302724.0248

DK Gandhi, R Gupta. Paediatric Neurology, Birmingham Children's Hospital, Birmingham, $U K$

Introduction Glucose transporter-1 (GLUT1) deficiency syndrome (OMIM \#606777) is an autosomal dominant condition resulting in reduced glucose transport into the brain. GLUT1 deficiency syndrome was first described in 1991 by De Vivo et al. The diagnostic finding is a low glucose concentration in the cerebrospinal fluid (CSF; mean 1.7 [SD $0.3 \mathrm{mmol} / \mathrm{L}$ ]) in the presence of normoglycaemia. GLUT1 deficiency syndrome can be confirmed by mutation analysis of the SLC2A1 gene. The spectrum is ever expanding with new mutations as also varying presentations. We present one such novel mutation.

Case report A Caucasian male was referred to the neurology department with global developmental delay, head nods, seizures and excessive daytime sleepiness. Over the years he had many investigations including repeated MRIs and EEGs and investigations for conditions with progressive myoclonic epilepsy. At the age of 14 years the parents gave a history of food intake reducing his head nods and other seizure types. He was then investigated for possible GLUT1 deficiency.

Results The initial investigations revealed a CSF sugar of 2.4 when the blood sugar was 5.9 (ratio of 0.4 ) which was low but not low enough for GLUT1 deficiency. Genetic testing revealed a mutation in the Exon 5 of the SLC2A gene c.647T>C (p.lle216Thr) not previuosly reported.

Conclusion GLUT1 deficiency should be suspected in a child with developmental delay, epilepsy and movement disorder. Novel mutations can result in the condition. Our case is one such example for novel mutation as well as refusal of ketogenic diet from late diagnosis.

\section{CEREBRAL PALSY AND CHEMOKINE CCL18 POLYMORPHISM IN VERY PRETERM INFANTS}

doi:10.1136/archdischild-2012-302724.0249

${ }^{1} \mathrm{H}$ Kallankari, '1 Huusko, 'T Kaukola, 'M Ojaniemi, ${ }^{2} \mathrm{SF}$ Kingsmore, 'M Hallman. ${ }^{1}$ Department of Paediatrics, Institute of Clinical Medicine, University of Oulu, Oulu, Finland; ${ }^{2}$ National Center for Genome Resources, Santa Fe, NM, USA

Background Cerebral palsy (CP) is a nonprogressive motor impairment syndrome caused by damage in the developing brain and it reveals clustering to preterm infants. Recently, genetic factors have been suggested as risk modifiers for CP. However, the individual genes causing predisposition to $\mathrm{CP}$ are still poorly understood. Low cord blood levels of CCL18 have been found to associate with CP in preterm infants. Since CCL18 gene is restricted to primates, it may be considered as a candidate for functionality in human brain.

Aims To investigate the association between the CCL18 gene single nucleotide polymorphisms (SNPs) and the cord blood levels of CCL18. Further to study the association between the CCL18 SNPs and the susceptibility to CP.

Methods A prospective cohort consisted of 161 children born very preterm (gestation < 32 weeks) in Oulu University Hospital during 1997-2006. Concentration of the cord blood CCL18 was analysed $(\mathrm{n}=99)$. Five CCL18 SNPs (rs1102934, rs2015086, rs2015070, rs2735835, rs712044) were genotyped. Cerebral palsy was confirmed at 5 years of age.

Results Two CCL18 SNPs associated with CCL18 ( $\mathrm{P}=0.011$; $\mathrm{P}=0.039$ ). Additionally, CCL18 (SNP rs2735835) associated with CP. Thus, CP occurred in 11 (18\%) of 61 children with GG genotype compared with $6(6 \%)$ of 100 children with AA/GA genotype (OR 4.1; $95 \%$ CI 1.3-12.5, $\mathrm{P}=0.013$ ).

Conclusions Variation of the CCL18 gene associates with CCL18 concentration and with predisposition to $\mathrm{CP}$ in very preterm infants. This is consistent with the hypothesis that CCL18 has a role in the complex sequence leading to brain damage.

\section{SEASONALITY OF CONGENITAL ANOMALIES IN EUROPE}

doi:10.1136/archdischild-2012-302724.0250

JM Luteijn, H Dolk, EUROCAT Working Group. EUROCAT Central Registry, Institute of Nursing Research, School of Nursing, University of Ulster, Jordanstown, UK

Investigation of seasonal patterns of congenital anomalies (CAs) can help identify environmental risk factors. Smaller datasets and spatiotemporal variation in previous studies have lead to conflicting evidence. The aim of our study was to investigate previously described associations and to generate new hypotheses on the role of seasonal factors in the etiology of CAs using a large high quality European dataset.

The European Surveillance for Congenital Anomalies (EUROCAT) is a European network of standardized population-based registries for the surveillance of CAs. A 2000-2009 dataset involving 19 EUROCAT registries and over 63,000 CAs was utilized to investigate seasonal patterns. Analysis of EUROCAT defined CAs was 